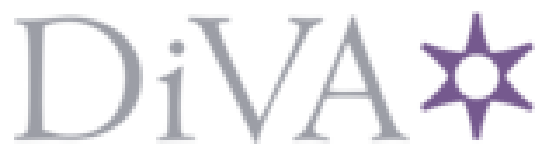

http://www.diva-portal.org

This is the published version of a paper published in .

Citation for the original published paper (version of record):

Aronsson, M. (2018)

La réception de Faïza Guène en Suède: la banlieue française en traduction

TTR: Traduction, Terminologie, Rédaction. Études sur le texte et ses transformations, 31(1): $97-125$

Access to the published version may require subscription.

N.B. When citing this work, cite the original published paper.

Permanent link to this version:

http://urn.kb.se/resolve?urn=urn:nbn:se:du-29734 


\section{TTR}

Traduction, terminologie, rédaction

\section{La réception de Faïza Guène en Suède : la banlieue française en traduction}

\section{Mattias Aronsson}

Traduire la banlieue : problématiques, enjeux, perspectives

Translating the Banlieue: Issues, Challenges, Perspectives

Volume 31, numéro 1, 1er semestre 2018

URI : https://id.erudit.org/iderudit/1062548ar

DOI : https://doi.org/10.7202/1062548ar

Aller au sommaire du numéro

Éditeur(s)

Association canadienne de traductologie

ISSN

0835-8443 (imprimé)

1708-2188 (numérique)

Découvrir la revue

Citer cet article

Aronsson, M. (2018). La réception de Faïza Guène en Suède : la banlieue française en traduction. TTR, 31 (1), 97-125. https://doi.org/10.7202/1062548ar
Résumé de l'article

L'article traite de la réception de l'oeuvre de Faïza Guène en Suède. Le corpus est composé de comptes rendus écrits par des critiques littéraires et publiés dans la presse entre 2006 ('’année de la première traduction de Guène en suédois) et 2016, ainsi que de comptes rendus rédigés par des blogueurs et publiés sur Internet pendant la même période. Nous examinons les thèmes les plus importants de cette réception ainsi que les références littéraires présentes dans les comptes rendus du corpus. Comme l'objectif du compte rendu d'une oeuvre traduite est de présenter un auteur étranger à un nouveau public, il est intéressant d'étudier les stratégies employées par les critiques : dans quels contextes littéraires et sociaux choisissent-ils d'inscrire Faïza Guène? Quels sont les arguments et références utilisés? Quelles différences y a-t-il entre les comptes rendus publiés dans la presse papier et ceux publiés dans la blogosphère? Les résultats montrent que les thèmes récurrents dans la réception suédoise incluent le succès de Kiffe kiffe demain en France, l'humour présent dans les textes de Guène et le milieu socioculturel dans lequel se déroulent ses récits. Les critiques littéraires discutent souvent du style, des dialogues et des portraits des personnages principaux dans les romans de l'auteure, et les avis sont divisés en ce qui concerne ces aspects de son écriture. Les références à d'autres écrivains associent l'oeuvre de Guène aux genres de la littérature migrante, de la littérature jeunesse et, à un moindre degré, de la littérature prolétarienne. Les opinions des blogueurs ne se distinguent pas de manière significative de celles exprimées dans la presse papier, mais le ton employé dans la blogosphère s'avère parfois plus subjectif que celui que l'on rencontre dans les comptes rendus traditionnels.
Ce document est protégé par la loi sur le droit d'auteur. L'utilisation des services d'Érudit (y compris la reproduction) est assujettie à sa politique d'utilisation que vous pouvez consulter en ligne.

https://apropos.erudit.org/fr/usagers/politique-dutilisation/ 


\title{
La réception de Faïza Guène en Suède : la banlieue française en traduction
}

\author{
Mattias Aronsson
}

Université de Dalarna

\begin{abstract}
Résumé
L'article traite de la réception de l'œuvre de Faïza Guène en Suède. Le corpus est composé de comptes rendus écrits par des critiques littéraires et publiés dans la presse entre 2006 (l'année de la première traduction de Guène en suédois) et 2016, ainsi que de comptes rendus rédigés par des blogueurs et publiés sur Internet pendant la même période. Nous examinons les thèmes les plus importants de cette réception ainsi que les références littéraires présentes dans les comptes rendus du corpus. Comme l'objectif du compte rendu d'une œuvre traduite est de présenter un auteur étranger à un nouveau public, il est intéressant d'étudier les stratégies employées par les critiques : dans quels contextes littéraires et sociaux choisissent-ils d'inscrire Faïza Guène? Quels sont les arguments et références utilisés? Quelles différences y a-t-il entre les comptes rendus publiés dans la presse papier et ceux publiés dans la blogosphère? Les résultats montrent que les thèmes récurrents dans la réception suédoise incluent le succès de Kiffe kiffe demain en France, l'humour présent dans les textes de Guène et le milieu socioculturel dans lequel se déroulent ses récits. Les critiques littéraires discutent souvent du style, des dialogues et des portraits des personnages principaux dans les romans de l'auteure, et les avis sont divisés en ce qui concerne ces aspects de son écriture. Les références à d'autres écrivains associent l'œuvre de Guène aux genres de la littérature migrante, de la littérature jeunesse et, à un moindre degré, de la littérature prolétarienne. Les opinions des blogueurs ne se distinguent pas de manière significative de celles exprimées dans la presse papier, mais le ton employé dans la blogosphère s'avère parfois plus subjectif que celui que l'on rencontre dans les comptes rendus traditionnels.
\end{abstract}

Mots-clés : étude de réception, Faïza Guène, compte rendu, presse papier, blogosphère

\section{Abstract}

The article examines the reception of Faiza Guène's works in Sweden. The corpus is made up of reviews written by literary critics and published in 
the press between 2006 (the year Guène's first novel was translated into Swedish) and 2016, as well as reviews written by bloggers and published on the Internet during the same period. The most important themes in this reception are examined, as are the literary references used in the articles. Since reviews of a translated work aim to introduce a foreign author to a new audience, it is interesting to examine the strategies of the reviewers: is Faïza Guène associated with a particular literary or social context? What arguments and references are used? What differences can be observed between reviews published in the printed press and those published in the blogosphere? The results show that the most common themes in the Swedish reception include the success of Kiffe kiffe demain in France, the humour in Guène's texts, and the sociocultural settings of her novels. The reviewers often discuss the style, the dialogues, and the portraits of the main literary characters in the author's novels - and there are mixed opinions regarding these aspects of her writing. The references made to other writers associate Guène's work with literary genres such as migrant literature, young adult fiction and, to a lesser extent, proletarian literature. Bloggers' opinions do not differ in any significant way from those expressed in the printed press, but the tone used in the blogosphere is sometimes more subjective than that of traditional literary reviews.

Keywords: reader-response study, Faïza Guène, literary review, printed press, blogosphere

\section{Introduction}

L'article traite de la réception suédoise de l'œuvre de Faïza Guène. L'auteure a publié son premier roman, Kiffe kiffe demain, en 2004. Il a été traduit en suédois deux ans plus tard sous le titre Kiffe kiffe imorgon. La traduction est apparue sur le marché à une époque où les expériences de la deuxième génération d'immigrés ont commencé à être représentées dans la littérature suédoise, un fait qui n'est pas passé inaperçu dans les médias ${ }^{1}$. C'est aussi l'époque où l'accès à Internet s'est démocratisé en Suède, et la blogosphère est devenue une arène alternative pour la critique littéraire (Steiner, 2015, p. 217). Pour cette raison, en entamant notre étude, nous avons assemblé un corpus de comptes rendus publiés d'une part dans la presse papier et, d'autre part, dans la blogosphère, entre 2006 (l'année de publication de Kiffe kiffe imorgon en Suède) et 2016. Le corpus a été établi en effectuant des recherches sur le nom de l'auteure et les titres des romans traduits, dans la base de

1. Citons à titre d'exemples de ce phénomène les œuvres de Leiva Wenger (2001), Daniel Boyacioglu (2003), Hassen Khemiri (2003) et Marjaneh Bakhtiari (2005). 
données Mediearkivet (pour la presse traditionnelle) et à l'aide du moteur de recherche Google (pour les blogues). Le corpus contient 54 articles et billets (voir l'annexe) et a été constitué en juin 2017. Les comptes rendus publiés dans la presse papier sont extraits de la presse nationale (Svenska Dagbladet, Dagens Nybeter, Aftonbladet, Expressen) et régionale (Göteborgs-Posten, Sydsvenskan, Östgöta Correspondenten, Borås Tidning, Upsala Nya Tidning, entre autres). Les comptes rendus publiés par les blogueurs ont été trouvés sur des sites web ou des blogues personnels tels que Bokhora, Bokmania et Ennannansidabok.

On constate d'emblée que la réception de Faïza Guène est dominée par les femmes. En effet, nous avons repéré dans le corpus 38 prénoms féminins et 9 prénoms masculins. Dans 8 cas, le sexe du commentateur nous est inconnu (il s'agit en l'occurrence de textes collectifs, de textes non signés ou de textes signés par un pseudonyme $)^{2}$.

Une fois le corpus assemblé, nous nous sommes penché sur les questions suivantes : quels sont les thèmes le plus souvent abordés dans la réception de l'œuvre de Faïza Guène en Suède? Quelles sont les informations fournies sur le roman et son auteure? Quelles sont les opinions exprimées dans les comptes rendus, et quels sont les arguments et références utilisés pour soutenir ces opinions? $\mathrm{Y}$ a-t-il des différences entre les comptes rendus publiés dans la presse traditionnelle et ceux publiés dans la blogosphère? Nous avons aussi voulu savoir s'il existait, dans les comptes rendus, des commentaires portant spécifiquement sur la traduction. Comme nous l'avons montré dans une étude précédente (Aronsson, 2015), la prose de Faïza Guène pose certains problèmes au traducteur suédois. Les difficultés sont principalement liées au vocabulaire familier et argotique du texte source, notamment le verlan, qui n'a

2. La situation s'explique sans doute en partie par une prépondérance correspondante des femmes dans le lectorat en général: selon l'association des éditeurs suédois Svenska förläggareföreningen (qui cite les chiffres de Mediebarometern, une enquête sur la consommation culturelle et médiatique en Suède menée depuis les années 1970), $41 \%$ des femmes lisaient quotidiennement un livre en 2016, comparativement à $29 \%$ des hommes (Svenska förläggareföreningen, 2017, n.p.). Cette tendance est encore plus marquée en ce qui concerne les œuvres écrites par des femmes. Qui plus est, les femmes dominent largement parmi les blogueurs (Findahl, 2013, p. 35). La même prépondérance féminine a d'ailleurs été observée dans une étude antérieure portant sur la réception de Marguerite Duras en Suède (Aronsson, 2016, p. 7-9). 
pas d'équivalent en suédois. Avant de répondre à ces questions, nous présenterons le cadre général de notre étude, c'est-à-dire le marché du livre en Suède, en nous concentrant sur les œuvres traduites.

\section{Le marché du livre en Suède}

Un trait distinctif du marché du livre suédois est son ouverture à l'extérieur (Lindqvist, 2005, p. 27-30). Il en résulte que la proportion de traductions parmi le nombre total de livres publiés est particulièrement importante. Ainsi, pendant la période étudiée par Lindqvist, c'est-à-dire de 1980 à 1994, 59 \% des textes littéraires publiés sur le marché suédois ont été des traductions, comparativement à $41 \%$ de textes originaux en suédois (ibid., p. 28).

Cette domination des traductions s'explique par le fait que la Suède est un petit pays semi-périphérique où les influences culturelles, intellectuelles et littéraires arrivent le plus souvent de l'étranger. Ainsi, le paysage littéraire de ce pays a longtemps été dominé par les traductions. Pendant le $\mathrm{XIX}^{\mathrm{e}}$ siècle, la majorité (50 à $70 \%$ ) des livres commercialisés en Suède ont été des traductions (Steiner, 2015, p. 94). Or, cela ne veut pas dire que l'accès au marché suédois soit facile pour tous les auteurs étrangers, quelle que soit leur origine culturelle et linguistique. Au contraire, l'ouverture du marché suédois concerne surtout, de nos jours, les œuvres anglo-américaines. Les chiffres officiels de la bibliographie nationale publiés par la bibliothèque royale (Kungliga Bibliotekets Nationalbibliografi) montrent en effet que la proportion des traductions de l'anglais dans le nombre total de traductions tourne actuellement autour de $70 \%$ (voir le tableau 1). On peut constater que la littérature traduite du français ne représente qu'une partie minime du marché du livre en Suède. Les titres «français» offerts sur le marché représentaient, pendant la période 2014-2016, entre $5,6 \%$ et $6,3 \%$ de toutes les traductions.

Le marché littéraire en Suède est ouvert à l'extérieur, certes, mais il est dominé par les écrivains anglo-américains. Pour un auteur de langue française, l'accès au marché peut être beaucoup plus difficile, car la part laissée aux écrivains non anglophones reste limitée. Dans les circonstances, il est intéressant de noter que tous les romans de Faïza Guène publiés à ce jour ont été traduits en suédois: Kiffe kiffe demain (Hachette, 2004)/Kiffe kiffe imorgon (Norstedt, 2006); Du rêve pour les oufs (Hachette, 2006)/Drömmar för dårar (Norstedt, 2008); Les gens du Balto (Hachette, 2008)/Sista 
beställningen på Balto (Norstedt, 2010); Un homme, ça ne pleure pas (Fayard, 2014)/En riktig man gråter inte (Norstedt, 2015). Ces romans ont été traduits par la même traductrice, Lotta Riad, et publiés par la même maison d'édition, Norstedt ${ }^{3}$.

Tableau 1. Traductions de livres en Suède (2014-2016) ${ }^{4}$

\begin{tabular}{|l|l|l|l|}
\hline Année & 2014 & 2015 & 2016 \\
\hline $\begin{array}{l}\text { (a) Nombre total de traductions } \\
\text { (toutes langues confondues) }\end{array}$ & 2735 & 2778 & 2537 \\
\hline $\begin{array}{l}\text { (b) Nombre (et pourcentage) } \\
\text { de traductions de l'anglais }\end{array}$ & $\begin{array}{l}1913 \\
(69,9 \%)\end{array}$ & $\begin{array}{l}1848 \\
(66,5 \%)\end{array}$ & $\begin{array}{l}1782 \\
(70,2 \%)\end{array}$ \\
\hline $\begin{array}{l}\text { (c) Nombre (et pourcentage) } \\
\text { de traductions du français }\end{array}$ & $\begin{array}{l}165 \\
(5,6 \%)\end{array}$ & $\begin{array}{l}175 \\
(6,3 \%)\end{array}$ & $\begin{array}{l}142 \\
(6,0 \%)\end{array}$ \\
\hline
\end{tabular}

La bonne circulation de l'œuvre de Faïza Guène en Suède est sans doute due au fait que Kiffe kiffe demain, le premier roman de l'auteure, a connu un véritable succès commercial à sa publication en France en 2004, un fait qui, comme nous le verrons plus loin, est souvent souligné dans les comptes rendus de notre corpus.

\section{La réception de Faïza Guène en Suède : thèmes récurrents}

Les thèmes abordés ci-dessous incluent le succès de Kiffe kiffe demain en France (2.1), le cadre des récits, c'est-à-dire la banlieue défavorisée (2.2), l'humour de l'auteure (2.3), les portraits des personnages principaux (2.4), le style, le vocabulaire et les dialogues (2.5), les références littéraires (2.6) et la traduction (2.7). À côté de ces thèmes qui sont les plus représentés dans le corpus, les critiques abordent d'autres enjeux tels que les conflits de générations, l'émancipation féminine et l'intégration des immigrés extra-européens dans les sociétés européennes. Ceux-ci ne seront cependant pas traités dans le présent article.

3. Norstedts bokförlag est la plus ancienne maison d'édition en Suède; elle a été fondée en 1823. Il s'agit d'un éditeur généraliste qui fait partie des acteurs dominants du marché de l'édition de ce pays.

4. Les chiffres proviennent de Nationalbibliografin i siffror (2016, p. 14). Le tableau 1 regroupe les livres imprimés de plus de 48 pages. La définition d'un « livre» comme étant une publication de plus de 48 pages est celle de l'UNESCO, qui est souvent utilisée au niveau international (voir par exemple Escarpit, 1970, p. 274). 


\subsection{Le succès de Kiffe kiffe demain en France}

Le succès du premier roman de Faïza Guène, Kiffe kiffe demain, sur le marché français est un aspect souvent mentionné dans les comptes rendus que nous avons étudiés. Les critiques suédois donnent des chiffres de vente (variant entre 225000 et 500000 exemplaires) et, pour accentuer le triomphe de cet ouvrage, ils mentionnent le nombre de langues dans lesquelles il a été traduit (entre 22 et 26). Le deuxième roman, Drömmar för dårar, est présenté comme «la continuation du succès de Kiffe kiffe demain» (Johansson, 2008, n.p. $)^{5}$, et Tunbäck-Hanson évoque «le succès éclatant» (2008, n.p.) du premier roman de Faïza Guène en $2004^{6}$. Le terme suédois succé («succès») à propos de Kiffe kiffe demain continue d'ailleurs d'être utilisé dans les comptes rendus d'En riktig man gråter inte en 2015 (v. Carlander, 2015 et Lindqvist, 2015). Cet aspect de la réception est sans doute lié à ce que Genette (1987, p. 268-270) appelle la «fonction informative» du commentateur. Mais il s'agit aussi d'un argument de vente, car les critique littéraires participent au processus de valorisation et de marketing des ouvrages qu'ils commentent ${ }^{7}$. Ceci vaut également pour les blogueurs, qui contribuent aussi à faire valoir l'ouvrage discuté. À titre d'exemple, on peut consulter Lindbäck (2006) et Petra (2009), qui ont évoqué le succès de Kiffe kiffe demain sur leurs blogues.

Dans ce contexte, on peut faire référence aux études de Jenkins (2006) et Jenkins, Ito et Boyd (2015) sur la «culture de participation» (participatory culture) $)^{8}$, qui implique que l'individu nest plus obligé de consommer passivement des produits culturels.

5. La citation en langue originale se lit comme suit : "uppföljaren till succén Kiffe kiffe imorgon». La traduction française est de nous. Il en sera de même de toutes les autres traductions qui suivront et pour lesquelles la citation originale en suédois est mentionnée en note.

6. «År 2004 gjorde Faïza Guène, nitton är gammal, dundersuccé med den lilla romanen Kiffe kiffe imorgon.»

7. Genette distingue la fonction informative (c'est-à-dire le fait de fournir des informations au lecteur potentiel) de la fonction de recommandation (le fait de lui recommander l'ouvrage). Un aspect intéressant dans ce contexte est que Genette attribue ces deux fonctions au préfacier. Or, à notre avis, le critique littéraire, dans son rôle d'intermédiaire entre auteur et lecteur, participe lui aussi souvent à ce double jeu d'information et de recommandation.

8. Jenkins définit la culture de participation ainsi : «Culture in which fans and other consumers are invited to actively participate in the creation and circulations of new content» (2006, p. 331). 
Comme nous l'avons montré dans une étude antérieure (Aronsson, 2016), les consommateurs ont aujourd'hui la possibilité de devenir des producteurs grâce à l'interactivité que leur offre Internet; on les appelle souvent prosommateurs (mot-valise formé de "producteur» et "consommateur») $)^{9}$. Les blogueurs de notre corpus jouent ce rôle de prosommateurs lorsqu'ils discutent des œuvres littéraires et en publient des comptes rendus. En même temps, ils participent à la commercialisation des œuvres dont ils discutent en attirant l'attention des internautes sur celles-ci. Du point de vue des éditeurs, il s'agit d'une promotion gratuite. Elle est considérée comme importante par les acteurs de la nouvelle économie du commerce électronique, car, très souvent, les internautes sont plus attentifs aux recommandations des blogueurs qu'aux opinions exprimées par les journalistes professionnels - pour ne pas parler des messages publicitaires traditionnels ${ }^{10}$. Ainsi, les avis et conseils des blogueurs sont désormais de première importance pour beaucoup d'entreprises commerciales, y compris les maisons d'édition.

\subsection{La banlieue}

Le thème de la banlieue est presque toujours mentionné dans les comptes rendus relatifs aux romans de Faïza Guène. Cette dernière est souvent présentée au public suédois comme une auteure qui a grandi elle-même dans une cité défavorisée - et plusieurs critiques insistent sur le fait qu'elle habite toujours en banlieue au moment de la publication de l'article (v. Tunbäck-Hanson, 2008 et Krönlein, 2010). On remarque qu'elle a souvent été appelée «förortens röst» et «en röst från förorten» [une voix de la banlieue] (Karlsson, 2010; Gordan, 2010), et le fait que ses parents soient des immigrés algériens est aussi mis en avant (Kopra, 2006; Risberg, 2007; Tunbäck-Hanson, 2008; Ekelund, 2013). Liés à cette thématique sont les thèmes de la précarité et de l'exclusion sociale ainsi que le manque de capital économique et culturel des personnages principaux de ses romans (Kopra, 2006; Bonnevier, 2010; Hagnell, 2015).

9. Le terme anglo-américain qui a servi d'inspiration est prosumer. Il a été utilisé dans de nombreuses études, dont celles de Ritzer (2010, p. 61-79) et Ritzer, Dean et Jurgenson (2012, p.379-398). Pour une discussion portant sur l'équivalent suédois (prosument), voir Steiner (2012, p. 61).

10. Voir Ritzer, Dean et Jurgenson, qui affirment : «Those who prosume on the Internet, especially Web 2.0, are very attractive to capitalists» (2012, p. 386). 
On peut noter que ces comptes rendus reprennent les clichés les plus répandus (banlieue, immigration, exclusion sociale, précarité). Il faut toutefois souligner qu'il s'agit parfois de commentaires portant sur la réception antérieure (suédoise et/ou française), que le journaliste tâche alors de problématiser. Citons notamment Gordan, qui critique la tendance à appeler l'auteure «la voix de la banlieue» :

Une voix de la banlieue - c'est une épithète qui me rend toujours méfiante. [...] le lecteur s'attend à ce qu'il y aura des bandes, du béton et de l'argot des cités - tout en continuant sa vie tranquille dans un tout autre univers social. Il y a ainsi un exotisme condescendant, qui se tape sur l'épaule, «maintenant je vais vous écouter, vous là-bas, voyez comme je suis bienveillant». Le terme établit une différence, une distance. Comme s'il était trop compliqué et trop menaçant de dire tout simplement: Un être humain parle d'un endroit. Il y raconte une histoire. (Gordan, 2010, n.p.) ${ }^{11}$

Ainsi, dans les comptes rendus étudiés, Faïza Guène est présentée au public suédois comme une auteure de la banlieue - et parfois comme la porte-parole censée représenter cette banlieue défavorisée et les gens qui y habitent. Toutefois, certains critiques notent que cette réception est stéréotypée et problématique.

\subsection{L'humour}

L'humour est une qualité qui est souvent mise en avant dans les commentaires portant sur les ouvrages de Faïza Guène, et les critiques suédois ont souligné cet aspect pour tous les romans de l'auteure. Kiffe kiffe imorgon a entre autres été appelé «un best-seller chaleureux et humoristique» (Falkehed, 2008, n.p. $)^{12}$. Concernant le deuxième roman, on trouve dans le corpus des affirmations telles que «Du rêve pour les oufs est un roman drôle, avec un humour exquis et sec» (Kvist, 2008, n.p.) et "Ahlème [la narratrice] est dotée d'un humour merveilleux»(Tunbäck-Hanson, 2008, n.p. $)^{13}$.

11. «En röst från förorten - det är ett epitet som alltid gör mig misstänksam. [...] nu blir det gäng och betong och förortsslang, förväntar sig läsaren, som själv lever tryggt på helt andra platser. Dels finns det därmed en förminskande exotism som liksom klappar sig själv på axeln, nu ska jag lyssna på er där borta, se så förstående jag är. Begreppet etablerar en skillnad, ett avstånd. Som vore det alltför komplicerat och hotande att bara säga: En människa talar från en plats. Där berättar hon en historia.»

12. «bästsäljande värme och humor.»

13. «Drömmar för dårar är [...] en rolig bok, med en skön torr humor» et «Ahlème är försedd med en underbar humor.» 
L'humour de l'auteure est mentionné aussi dans les comptes rendus de Sista beställningen på Balto (v. Svensson et Gourdon, 2010; Karlsson, 2010). Et pour ce qui est d'En riktig man gråter inte, les critiques persistent à accentuer le même thème :

Avec un humour chaleureux et une joie de mots pétillante, elle peint un tableau passionnant et complexe de sa génération et des conditions dans lesquelles elle a grandi. (Chahboun, 2015, n.p.) ${ }^{14}$

Faïza Guène écrit avec humour, chaleur et un langage qui coule de source. (Carlander, 2015, n.p.) ${ }^{15}$

Un homme, ça ne pleure pas est un roman très charmant, qui décrit de l'intérieur la réalité des HLM. L'assimilation et l'exclusion se trouvent au centre de l'intérêt, et c'est raconté avec beaucoup d'humour et de chaleur. (Hagnell, 2015, n.p.) ${ }^{16}$

Nous constatons, en ce qui concerne le thème de l'humour, que la réception suédoise ressemble beaucoup à la réception française de Kiffe kiffe demain telle qu'elle s'est exprimée dans la blogosphère francophone (v. Aronsson, 2012, p. 69). Ainsi, les critiques littéraires et les blogueurs, les Français et les Suédois, semblent unanimes sur ce point : les romans de Faïza Guène sont drôles! En effet, nous n'avons pas trouvé une seule voix exprimant le contraire.

\subsection{Les portraits des personnages}

La réception de l'œuvre de Faïza Guène s'avère mixte en ce qui concerne les portraits des personnages principaux dans les romans. Certains critiques louent ces portraits, les jugeant crédibles, nuancés et complexes, tandis que d'autres les trouvent banals et trop simplifiés. Voici, à titre d'exemple, quelques voix divergentes portant sur les portraits dans En riktig man grăter inte:

Avec grand amour et une attention particulière aux détails, la jeune auteure à succès Faïza Guène peint un portrait tendre d'une famille algérienne aux alentours de Nice, avec un père chaleureux et entêté un caractère fort! - une mère colérique et possessive, et trois enfants qui prennent tous des chemins différents dans la vie. Avec tous ces petits détails, les personnages ne deviennent pas seulement vivants,

14. «Med varm humor och sprudlande ordglädje tecknar hon en medryckande och komplex bild av sin generations uppväxtvillkor.»

15. «Faïza Guène skriver med humor, värme och ett friskt flödande språk.»

16. «En riktig man gråter inte är en alldeles förtjusande roman, skildrad inifrån hyreskasernernas verklighet. Assimilering och utanförskap står i centrum, berättat med stor humor och värme.» 
mais aussi crédibles. C'est une histoire racontée par une écrivaine qui connaît bien l'univers quelle décrit. (Hagnell, 2015, n.p.) ${ }^{17}$

Le problème, c'est les portraits simplifiés des personnages, les stéréotypes dépourvus de toute forme d'ironie. (Eriksdotter Andersson, 2015 , n.p. $)^{18}$

Avec son langage vivant, plein d'anecdotes et d'humour, elle peint un peu maladroitement les différences culturelles qui deviennent kitsch, et les personnages autour de Mourad [le personnage principal] ressemblent à des clichés au lieu de portraits bien dessinés. (Strängberg, 2015, n.p.) ${ }^{19}$

En somme, Mourad paraît inachevé, incomplet : tantôt prétentieux, moralisant, vaniteux, tantôt intelligent et conciliant. (Setterwall Klingert, 2015, n.p. $)^{20}$

Il n'y a donc pas consensus en ce qui concerne les portraits des personnages. Les exemples ci-dessus sont tirés de la presse traditionnelle, mais la même hétérogénéité des évaluations apparaît dans la blogosphère. À propos de Doria, la narratrice de Kiffe kiffe imorgon, nous trouvons ces avis contradictoires:

La voix narrative de Doria dans Kiffe kiffe demain est forte et retentissante, elle est drôle, intelligente et elle vise juste (Eklund, 2009 , n.p. $)^{21}$.

C'est un peu énervant de constamment entendre parler de ses problèmes [...] Son passe-temps favori est de trouver des défauts chez autrui (9c läser Kiffe kiffe imorgon av den franska författarinnan Faïza Guène, 2012, n.p. ${ }^{22}$.

17. «Med stor kärlek och känsla för detaljer målar unga succéförfattaren Fä̈a Guène ömt ett porträtt av en algerisk familj i utkanten av Nice, med en egensinnig, varm pappa - en stark karaktär! - en kolerisk, omvårdande mamma och tre barn som alla utvecklas åt olika håll. Med de små detaljerna blir persongalleriet inte bara levande utan också trovärdigt. Detta är berättat av en författare som kan den värld hon skildrar.»

18. «Problemet är de enkla personteckningarna, de helt oironiska stereotyperna.»

19. «Hennes livfulla språk, anekdotiskt och humoristiskt, målar med bred pensel upp kulturskillnaderna som blir till kitsch och figurerna kring Mourad klichéer istället för utmejslade karaktärer.»

20. «Överhuvudtaget känns Mourad ofärdig, halvsmält: i ena stunden pretentiös, dömande, självgod, i nästa intelligent och försonande.»

21. «Dorias berättarröst i Kiffe kiffe imorgon är stark och självlysande, hon är rolig, smart och träffsäker.»

22. «Det är lite störande att hela tiden läsa om hennes problem [...] Hennes största intresse är att hitta fel hos andra.» 
Ainsi, les opinions diffèrent également dans la blogosphère, et les jugements sur la qualité des portraits ne sont pas unanimes. Alors que Drömmar för dårar est décrit comme un «bon roman qui mérite d'être lu» (Kristin, 2014, n.p.) et comme un texte transmettant «un message qu'il faut écouter» (Stjärtmes, 2009, n.p.), Virdelöv, pour sa part, s'exclame : «Bonjour les simplifications» $(2008 \text {, n.p. })^{23}$.

\subsection{Le style, le vocabulaire et les dialogues}

Le corpus examiné contient de nombreux commentaires sur le style de l'auteure, le vocabulaire qu'elle affectionne et la façon de parler de ses personnages. Le fait que Faïza Guène ait contribué à introduire l'argot des jeunes banlieusards dans le monde littéraire en France ne passe pas inaperçu :

Elle ne cherche pas non plus un langage novateur, mais son écriture est légère, colorée et truffée d'argot et d'arabe. (Pärssinen, 2008, n.p. $)^{24}$

La prose de Guène est laconique et non sentimentale, et elle capte parfaitement les différentes tonalités des discours. C'est un langage très riche qui exprime aussi bien l'argot des jeunes, les propos grossièrement sexistes, le bavardage raciste à moitié dissimulé, que les tentatives prétentieuses de bien parler. (Flakierski, 2010, n.p.) ${ }^{25}$

Pour ce qui est des dialogues, les avis sont divergents, comme c'était le cas pour les portraits des personnages. Certains critiques trouvent les dialogues très bien réussis tandis que d'autres soutiennent le contraire. Voici, à titre d'exemple, deux opinions sur les dialogues du second roman de Guène, Drömmar för dårar :

Toutefois, le dialogue est toujours un peu boiteux, avec des phrases guindées qui s'entassent d'une façon peu harmonieuse. (Filip Otelea, 2008 , n.p. $)^{26}$

[C'est une prose où] une critique sociale aiguë coexiste sans le moindre

23. «boken är bra och läsvärd»; «ett budskap som är värt att ta till sig» et «Välkommen till förenklandet.»

24. «Hon lägger inte heller krutet på ett nyskapande språk, utan skriver lätt och målande och tryfferar med slang och arabiska.»

25. «Guène skriver en korthuggen, osentimental prosa som tonsäkert fångar på kornet de olika idiomen, det är ett fantastiskt rikt språk som återger lika bra ungdomsslang, grovt sexistiskt mummel, förtäckt rasismsvammel som pretentiösa försök att tala fint.»

26. «Dialogen har dock fortfarande en tendens att halta, med styltiga meningar som staplas på varandra i en oharmonisk hög.» 
problème avec des dialogues amusants portant sur les feuilletons télé à la mode. Une langue qui, dans des images instantanées, peut capter l'embarras des immigrés et la détermination d'une jeune personne : j'ai l'intention de me construire une langue, ici, dans ce nouveau pays. (Adolfsson, 2008, n.p.) ${ }^{27}$

À notre avis, il n'est pas surprenant que les critiques expriment des avis contraires concernant les dialogues dans les romans de Guène étant donné qu'une telle divergence a été identifiée aussi pour les portraits des personnages dans cette œuvre. En effet, les deux aspects (portraits et dialogues) nous semblent très liés : l'aspect physique et moral d'un personnage fictif, ses gestes, ses actions, ses paroles et sa façon de s'exprimer, contribuent tous à la représentation littéraire du personnage en question. Ainsi, si on manque de consensus concernant la qualité des portraits, il est logique que les opinions diffèrent aussi pour ce qui est des dialogues.

\subsection{Les références littéraires}

Quand un critique littéraire présente un auteur étranger qui n'est pas (ou qui est peu) connu dans le pays cible, il peut se référer à des figures connues dans le milieu visé, pour faciliter la compréhension du public. En l'occurrence, les références littéraires récurrentes relevées dans notre corpus incluent les auteurs suédois Marjaneh Bakhtiari et Jonas Hassen Khemiri (voir les comptes rendus de Lindbäck, 2006; Lunderquist, 2008; Adolfsson, 2008; Bonnevier, 2010 et Nilsson, 2010). Ces deux écrivains ont fait leurs débuts peu avant la première traduction de Faïza Guène en langue suédoise. Leurs premiers romans (Hassen Khemiri, 2003; Bakhtiari, 2005) abordent en partie les mêmes thèmes que les récits de Guène : les expériences des adolescents issus de la deuxième génération d'immigrés extra-européens et leur intégration, parfois difficile, dans la société occidentale ${ }^{28}$. Qui plus est, l'humour constitue aussi un trait saillant des romans de Bakhtiari et d'Hassen Khemiri. Leurs noms sont donc évoqués pour que le lecteur du compte rendu puisse situer Faïza Guène dans le paysage littéraire de la Suède. Une cri-

27. «Där skarp samhällskritik smidigt löper samman med fyndiga dialoger om de senaste tevesåporna. Ett språk som i ögonblicksbilder kan fånga immigranternas kluvenhet, och en ung människas beslutsamhet: här, i det nya landet, tänker jag bygga mig ett språk.»

28. Il s'agit en l'occurrence des personnages principaux d'origine iranienne (chez Bakhtiari) et maghrébine (chez Hassen Khemiri). 
tique (Adolfsson, 2008) fait aussi référence à Susanna Alakoski et Åsa Linderborg, qui sont évoquées en tant que représentantes d'une «nouvelle génération» d'écrivains prolétariens. Toutes les deux ont publié des romans dans lesquels le thème de la précarité sociale est prédominant (Alakoski, 2006; Linderborg, 2007). La littérature prolétarienne, appelée arbetarlitteratur en Suède (littéralement "littérature des travailleurs»), occupe une place de premier ordre dans l'histoire littéraire de ce pays et elle a été dominante pendant l'apogée de la société industrielle, c'est-à-dire des années 1930 jusqu'aux années 1960. Après une période de silence, on parle dans les années 2000 de l'émergence d'une nouvelle génération d'auteurs prolétariens, parfois exemplifiée par les écrivaines susmentionnées.

Les références aux auteurs suédois dominent, mais on trouve dans le corpus des noms qui appartiennent à d'autres sphères culturelles. La culture française est bien sûr représentée : d'abord par les références à Françoise Sagan, qui a aussi débuté sa carrière littéraire à l'âge de 19 ans. Guène est appelée «la Françoise Sagan de la banlieue» (Svensson et Gourdon, 2010, n.p.) et «la Françoise Sagan des gratte-ciel»(Hagnell, 2015, n.p.) ${ }^{29}$. Le roman La vie devant soi de Romain Gary (Émile Ajar) est mentionné à deux reprises (Anonyme, 2009; Kristin, 2014) par des blogueurs. La littérature anglo-américaine est également évoquée, ce qui n'est pas étonnant si l'on considère l'ouverture de la Suède vers cette sphère culturelle. Le roman Jane Eyre de Charlotte Brontë (Adolfsson, 2008) ainsi que des ouvrages pour la jeunesse tels que Anne of Green Gables de Lucy Maud Montgomery (Adolfsson, 2008; Kjersen Edman, 2008) et The Secret Diary of Adrian Mole de Sue Townsend (Gordan, 2010) sont mentionnés dans deux comptes rendus de la presse papier et dans un texte de la blogosphère. Les personnages de Jane Eyre et Anne of Green Gables sont mis en parallèle avec Ahlème, l'héroïne du roman Du rêve pour les oufs (Adolfsson, 2008). Quant à Adrian Mole, il est cité comme un jeune personnage fictif ressemblant aux adolescents décrits par Guène : "[s]ouffrant de problèmes juvéniles, mais universaux, tels que boutons et amourettes, ils errent dans le pays limitrophe entre l'enfance et l'âge adulte»(Gordan, 2010) ${ }^{30}$.

29. «förortens Françoise Sagan» et «höghusens Françoise Sagan». On trouve la même référence dans la réception française de Kiffe kiffe demain (v. Olsson, 2011, p. 209; Aronsson, 2012, p. 69).

30. «[M]ed både ungdomliga problem i all deras allmänmänsklighet; finnar, vilsenheten i landet mellan barndom och vuxenhet, förälskelse». 
Nous notons, en général, que les références littéraires qui sont faites dans les comptes rendus associent Faïza Guène à une littérature représentant la deuxième génération d'immigrés dans la société occidentale, une catégorie de la population qui se trouve souvent au bas de l'échelle sociale. Ainsi, il y a dans le corpus un lien avec le champ de la migrant literature et la littérature contemporaine d'inspiration prolétarienne. Toutefois, on établit également une association avec la littérature jeunesse plus traditionnelle destinée aux jeunes adultes.

\subsection{La traduction}

À la publication du roman Du rêve pour les oufs en traduction suédoise en 2008, une critique littéraire a jugé que l'ouvrage était «brillamment traduit» (Adolfsson, 2008, n.p.) et un autre, que la traduction était "convaincante» (Lunderquist, 2008, n.p.) ${ }^{31}$. La qualité de la traduction du roman Les gens du Balto a également été reconnue :

Le langage des monologues est parfaitement nuancé en fonction du personnage qui parle et l'argot des banlieues, en particulier, est très bien traduit par Lotta Riad. (Nilsson, 2010, n.p.) ${ }^{32}$

Le ton des personnages est pointu, cru, et agressif - ici je veux prendre l'occasion de mentionner la traductrice Lotta Riad, qui a transmis les difficultés de l'argot français dans un équivalent suédois. (Gordan, 2010, n.p.) ${ }^{33}$

C'est un langage rapide qui montre une mauvaise attitude, et la traduction suédoise de Lotta Riad fait mouche. (Enander, 2010, n.p. $)^{34}$

On trouve en outre dans le corpus des éloges sur la traduction d'Un homme, ça ne pleure pas (Carlander, 2015). Il convient de noter que tous les exemples cités ci-dessus ont été repérés dans la presse papier; les blogueurs, pour leur part, commentent rarement

31. «glansfullt översatt» et «övertygande.»

32. «Språket i monologerna är skickligt nyanserade i enlighet med den som för talan, och särskilt förortsslangen har återgivits strålande av översättaren Lotta Riad.»

33. «Tonen hos karaktärerna är vass, rå och inställd på försvar - här vill jag passa på att nämna översättaren Lotta Riad som överfört den svåra franska slangen till svensk motsvarighet.»

34. «Det är ett rappt språk som lider av taskig attityd, och har skickligt och träffsäkert översatts till svenska av Lotta Riad.» 
la traduction ${ }^{35}$. Une exception à cette règle est le compte rendu de Pelleskopmatten (2014), qui contient des éloges semblables à ceux repérés dans la presse traditionnelle.

Ainsi, tous les critiques ayant formulé des commentaires sur la traduction estiment que les versions en suédois sont idiomatiques et réussies. Cependant, il n'y a aucune indication qu'ils ont consulté les textes sources pour juger de la qualité de la traduction. En effet, les aspects de la traduction qui sont discernables dans une étude contrastive (texte source - texte cible) ne sont jamais abordés. Ces aspects concernent notamment la normalisation des textes traduits, qui implique ici que le discours argotique et «beur» des personnages de Faïza Guène apparaît moins argotique et moins «beur» dans les textes cibles. Certains mots d'origine arabe du texte source («bled», «flouse », «maboul» et «walou», entre autres) sont traduits par des mots ayant une autre étymologie, ce qui rend la présence maghrébine moins visible dans le texte cible. Par exemple, le terme d'origine maghrébine "walou», utilisé dans Kiffe kiffe demain, est dans la traduction suédoise rendu par «nada», un terme espagnol. Un personnage secondaire du même roman, Aziz, est dit avoir un «accent de blédard» dans le texte source. Or, la connotation maghrébine est perdue dans la traduction suédoise, où il a simplement un «brytning» [accent étranger]. D'ailleurs, lorsque son discours est reproduit dans la version suédoise, l'accent en question ressemble beaucoup à un accent espagnol (Aronsson, 2015, p. 34$38)^{36}$. Cette présence espagnole dans le texte cible s'explique peutêtre par les flux d'immigration qui s'observent dans chacun des pays concernés. Une importante communauté maghrébine s'est installée en France depuis la Seconde Guerre mondiale, ce qui n'est pas le

35. Le résultat confirme ce qui a été observé dans la réception suédoise de l'œuvre de Marguerite Duras (Aronsson, 2016, p. 16). Les comptes rendus rédigés par les internautes sont souvent très succincts et vont "droit au but». Aux yeux des blogueurs, la question de la traduction n'est pas, de toute évidence, un sujet suffisamment important pour être discuté.

36. Bien sûr, l'orthographe adoptée pour reproduire son discours essaie d'imiter un discours oral. Walpole appelle ce procédé «eye dialect» et explique qu'il consiste à transformer l'orthographe standardisée pour illustrer une prononciation qui diffère de la norme : «[the author] can twist standard orthography to suggest nonstandard pronunciations» (1974, p. 192). En l'occurrence, l'accent étranger de ce personnage fictif (Aziz) le rend différent, un peu comique; il devient «l'Autre» par sa façon de s'exprimer : décidément maghrébin dans le texte de départ, plutôt hispanophone dans le texte cible. 
cas en Suède. Dans ce dernier pays, l'argot banlieusard a plutôt été influencé par les immigrés hispanophones venus de l'Amérique latine (v. Doggelito et Kotsinas, 2004, p. 9-10) ${ }^{37}$.

Le procédé de normalisation vaut aussi pour l'aspect argotique, qui paraît moins saillant dans la version suédoise. Par exemple, dans la version originale du roman $D u$ rêve pour les oufs, le discours des personnages est «traduit» en "français standard» au moyen de notes de bas de page, pour assurer la compréhension du lecteur implicite. Dans la traduction suédoise, le discours a été directement normalisé. Pour ne donner que deux illustrations du phénomène, l'expression "c'est cheum» est accompagnée, dans la version originale, d'une note de bas de page précisant que «cheum» veut dire «moche» en verlan, et l'adjectif verlanisé «scrède» est traduit par «discret» pour le lecteur français. Les termes utilisés dans la traduction suédoise, «taskigt» [vache, moche] et «diskret» [discret], ne présentent pour leur part aucune difficulté pour le lecteur et ne sont donc pas explicités par des notes (Aronsson, 2015, p. 44).

Un autre procédé fréquemment employé par la traductrice est celui de la compensation. La prose de Faïza Guène contient de nombreux traits d'oralité (par exemple, la chute de «ne» en tant que première partie des négations, parfois accompagnée par la disparition du sujet comme dans "y a pas», au lieu de "il n'y a pas»). Ces graphies imitant la langue parlée n'ont pas d'équivalents en suédois. En conséquence, l'oralité a souvent été transposée ailleurs dans le texte cible et exprimée par d'autres moyens : des mots tronqués «nån, nåt » pour «någon, något» [quelqu'un, quelque chose], «sen» pour «sedan» [puis], ou l'ajout de jurons par rapport au texte source (Aronsson, 2015, p. 40-41) (38. $^{38}$

37. Outre les immigrés venus de l'Amérique latine, un nombre important de travailleurs sont arrivés de la Finlande, la Grèce et la Turquie dans les années 1960 et 1970 . Plus récemment, la Suède a vu l'arrivée de beaucoup de réfugiés de guerre de la Bosnie, la Somalie, le Kurdistan, l'Afghanistan et la Syrie, pour ne nommer que quelques pays de départ. Par conséquent, les langues parlées par tous ces groupes d'immigrés ont influencé l'argot banlieusard en Suède.

38. Il n'est pas aisé de savoir pourquoi la traductrice a ajouté des jurons, ce qui est un choix un peu surprenant. L'hypothèse présentée dans notre étude antérieure (Aronsson, 2015, p. 41) est qu'elle part du principe que les "gros mots » suédois sont moins expressifs que les jurons français et que, par conséquent, il en faut un plus grand nombre dans le texte cible pour produire le même effet sur le lecteur. Toutefois, ceci n'est qu'une hypothèse. 
Un dernier détail au sujet du thème de la traduction : le nom de la traductrice (Lotta Riad) est presque toujours évoqué par les critiques et blogueurs, même dans les cas où il n'y a pas, dans le compte rendu, de remarques sur la qualité de la traduction. Sans être forcément bien connue du grand public, Lotta Riad possède un solide $\mathrm{CV}$, ayant traduit en suédois des auteurs tels que Emmanuel Carrère, Fatou Diome, Ying Chen et Hiner Saleem - ce qui montre une certaine prédilection pour la "littérature migrante» (migrant literature). D'ailleurs, son patronyme comporte un capital culturel important : elle est la sœur de Tomas Riad, professeur de langues scandinaves à l'Université de Stockholm et membre de l'Académie suédoise depuis 2011.

\section{Aspects complémentaires de la réception suédoise}

Les autres aspects de la réception de l'œuvre de Faïza Guène en Suède traités ici incluent l'usage didactique du roman Kiffe kiffe imorgon dans l'enseignement (3.1), les visites de Faïza Guène en Suède (3.2) et le style des blogueurs qui se sont exprimés à propos des ouvrages de l'auteure (3.3). Ces trois aspects ne sont pas directement liés aux questions de recherche de notre étude, mais ils se sont révélés particulièrement intéressants après une analyse approfondie du corpus.

\subsection{L'usage didactique de Kiffe kiffe imorgon}

Le roman Kiffe kiffe demain est écrit comme un journal intime rédigé par une narratrice adolescente. C'est peut-être la raison pour laquelle certains enseignants l'intègrent à leurs activités pédagogiques $^{39}$. Nous ignorons le nombre total d'enseignants suédois qui se sont servis d'un roman de Faïza Guène; nous ne pouvons présenter que les cas où le travail en classe a laissé des traces dans la blogosphère. Notre corpus montre que la traduction suédoise du roman a été utilisée à des fins didactiques par au moins deux enseignants - qui ont ensuite partagé et diffusé les travaux de leurs élèves sur Internet. Une classe de première au lycée a rédigé un compte rendu du roman sous forme de texte "wiki», c'est-à-dire un travail collaboratif dans lequel plusieurs rédacteurs contribuent au résultat final (SP2A Bolandgymnasiet, 2009). Le texte a été publié en ligne et il a été commenté par une bibliothécaire du

39. Notre étude portant sur la réception française de Kiffe kiffe demain sur Internet (Aronsson, 2012) avait déjà montré que ce roman a été utilisé dans les salles de classe en France. 
centre de documentation et d'information (CDI) du même lycée. Cela montre qu'il s'agissait d'un projet didactique ambitieux, dans lequel l'interaction dépassait la communication traditionnelle entre enseignant et élèves. En l'occurrence, l'enseignant a fait en sorte qu'il ne soit pas le seul lecteur du compte rendu. Cet aspect est particulièrement important, car l'existence d'un public cible concret c'est-à-dire des lecteurs réels à l'extérieur de la salle de classe - est un facteur de motivation important pour stimuler l'expression écrite des élèves (Tornberg, 2009, p. 179). Dans ce contexte, les technologies de communication fournies par Internet peuvent s'avérer très utiles pour établir les contacts nécessaires, un fait qui a été souligné dès le début des années 2000 par Brodow (2001, p. 122-140) et qui reste d'actualité (v. Dixon et Thomas, 2015).

Nous avons repéré dans le corpus examiné un second cas où une enseignante s'est servie de Kiffe kiffe imorgon, cette fois-ci dans une classe de collège (correspondant à la $3^{\mathrm{e}}$ dans le système français). L'enseignante a formulé des questions de réflexion sur le roman et les a déposées sur un blogue ( 9 c läser Kiffe kiffe imorgon av den franska författarinnan Faïza Guène, 2012). Les élèves ont ensuite répondu aux questions par écrit, en groupes de deux ou trois, avant de commenter les réponses des autres groupes sur le blogue. L'enseignante a ainsi créé une interaction du type peer feedback entre les élèves, pour encourager le travail collaboratif ${ }^{40}$. Par conséquent, elle s'est inspirée des principes pédagogiques de Lev Vygotsky. Dans cette tradition dite «socioculturelle» - qui est prédominante dans le système éducatif suédois -, on voit l'apprentissage avant tout comme un processus collectif (Säljö, 2015, p. 89-107).

On trouve aussi dans le corpus l'avis d'une bibliothécaire travaillant à la bibliothèque municipale d'une ville du sud du pays, qui estime que Kiffe kiffe imorgon est une «bonne représentation de la vie d'une jeune fille musulmane » et qui l'inclut dans la présentation de ses «livres favoris» dans la catégorie littérature pour la jeunesse (Ohlsson, 2016, n.p.) ${ }^{41}$. Il s'agit d'une liste d'œuvres proposées par

40. Les questions de réflexion formulées par l'enseignante se trouvent sur le site web, ainsi que les réponses écrites des élèves et le peer feedback que les élèves se sont donnés. Par conséquent, la structure de l'exercice est facilement repérable. Il faut souligner, cependant, que seul le résultat est visible pour le chercheur. Le processus de travail et la dynamique de la salle de classe ne le sont pas, car nous n'y étions pas présent pour observer l'interaction entre l'enseignante et les élèves. 41. «Bra skildring av en ung muslimsk flickas liv» et «favoriter i bokhyllan». 
la bibliothécaire pour inspirer et encourager les adolescents de cette ville à lire des textes littéraires. Il y a donc une idée didactique à la base du projet, nous semble-t-il.

Cependant, pour nuancer les choses en ce qui concerne le rôle de Kiffe kiffe imorgon en tant qu' "invitation à la lecture" pour les jeunes Suédois, il faut aussi évoquer un commentaire formulé dans la blogosphère par une personne qui semble aussi travailler dans le secteur de l'enseignement (au collège ou au lycée). À en juger par son témoignage, elle a essayé d'utiliser Kiffe kiffe imorgon avec ses élèves, mais sans succès :

[Le roman] ne leur a pas parlé du tout, ils ne comprennent pas l'ironie et l'autodérision [de la narratrice], ils sont trop occupés par la construction de leurs propres identités pour pouvoir s'identifier au personnage principal. Ils n'arrivent pas à décoder les différents niveaux de sens du texte. J'ai lu certains extraits à haute voix, p. ex. la scène où elle achète des serviettes hygiéniques (qui m’a fait marrer énormément), mais ils l'ont trouvée embarrassante. (Asalun, 2008, n.p. $)^{42}$

L'âge des élèves dans cet exemple n'est pas révélé et, en tant que lecteur du blogue, on ne connaît pas la raison pour laquelle le texte a manqué sa cible. Or, de nombreux experts de la sociologie de la littérature et des études de réception soulignent l'importance pour le lecteur de «se retrouver» dans le texte. Pour Sarland, par exemple, c'est un facteur essentiel pour les lecteurs adolescents. Dans un ouvrage intitulé Young People Reading: Culture and Response, il consacre un chapitre au thème de l'identification des lecteurs (Sarland, 1991, p. 79-90). De toute évidence, c'est cette identification personnelle de la part des élèves qui n'a pas eu lieu dans le cas discuté ci-dessus.

\subsection{Les visites de l'auteure en Suède}

En règle générale, il faut un événement d'actualité pour attirer l'attention des médias. La publication du nouveau roman d'un auteur connu peut constituer un tel événement. De fait, la majorité des articles de notre corpus portent sur la publication d'un nouvel

42. «Den har inte alls gått hem där, de förstår inte ironin och självdistansen, de är för mycket uppe i sina egna identitetsbyggen för att relatera till huvudpersonen. De kan inte läsa av de olika betydelselagren i texten. Har läst delar högt, bl.a. köpa-bindor-scenen (som jag har jättekul åt) och de tyckte bara att det var pinsamt.» 
ouvrage de Faïza Guène. À côté des comptes rendus critiques, on trouve toutefois quelques articles de presse consacrés aux visites de l'auteure en Suède pendant la période examinée (2006-2016). Elle y est passée pour la première fois en 2007 pour recevoir le prix Peter Pan - un prix suédois offert chaque année à un écrivain étranger, auteur d'un livre pour enfants ou de jeunesse traduit en suédois. Faïza Guène a reçu le prix pour Kiffe kiffe imorgon, ce qui a donné lieu à des articles dans la presse générale (Risberg, 2007; Steinsaphir, 2007). Ainsi, on constate que Kiffe kiffe imorgon a été reçu en Suède comme un livre jeunesse plutôt qu'un roman pour adultes (le prix en est un indice de même que l'usage didactique et les recommandations de la bibliothécaire dont il a été question).

Le lancement d'un nouveau livre s'accompagne généralement d'une tournée de promotion. Dans le marché du livre actuel, l'auteur est souvent sa propre marque de commerce, ce qui veut dire qu'il est le premier porte-parole de son ouvre et qu'il doit participer aux activités de commercialisation de ses ouvrages (entrevues, conférences de presse, lectures publiques, etc.). Or, Faïza Guène aurait fait une seule visite promotionnelle visant spécifiquement la Suède, et ce, à la publication de Sista beställningen på Balto en 2010. Des journalistes ont alors réalisé des entrevues avec elle (Krönlein, 2010; Persson, 2010), tandis que d'autres (Gordan, 2010; Svensson et Gourdon, 2010) ont annoncé qu'elle allait parler en public à Stockholm et à Malmö. La rareté des visites de l'auteure s'explique peut-être par le fait que la Suède est un marché périphérique peu rentable (au niveau des chiffres de vente potentiels).

\subsection{Les blogueurs}

Comme nous l'avons vu, les opinions des blogueurs ne diffèrent pas de manière significative de celles des critiques établis de la presse papier. Par contre, le style employé par ces «prosommateurs» se révèle parfois différent de celui que l'on rencontre dans la presse. Les critiques établis s'expriment dans un style journalistique traditionnel : leur discours est caractérisé par son caractère impersonnel, sa distanciation et son objectivité (ou pseudo-objectivité) ${ }^{43}$. Les

43. Voir notamment, au sujet de ce genre textuel, Möijer (1989, p.27-28), Carlsson (2010, p. 232) et Cassirer (2003, p. 158). Voir aussi notre étude sur la réception de Marguerite Duras en Suède, dans laquelle le même phénomène a été identifié (Aronsson, 2016, p. 13-15). 
blogueurs, eux, emploient parfois un ton beaucoup plus subjectif, voire «égocentrique». Voici deux exemples illustrant ce phénomène :

Je ne sais pas ce qu'il y avait dans ce livre qui m'a rebuté. J'ai senti quelque part que je n'arrivais jamais à établir une relation avec les personnages. Ils ne s'accrochaient pas bien et je n'arrivais pas à les distinguer l'un de l'autre. Cela a rendu la lecture un peu chaotique, pour ne pas dire ennuyeuse. J'avais du mal à distinguer les personnages et leurs rôles respectifs. Je pense qu'il faut lire certains livres à un moment précis pour pouvoir les apprécier. Ce livre-ci appartient peut-être à cette catégorie. (Malin, 2012, n.p.) ${ }^{44}$

La voix narrative, Doria 15 ans, me fait tout de suite penser au petit Momo dans un de mes livres favoris, La vie devant soi de Romain Gary. Cela me donne des sentiments mixtes. Je me réjouis à l'idée de lire quelque chose de semblable, en même temps, ce n'est pas facile de se mesurer à la hauteur d'un livre favori. (Kristin, 2014, n.p.) ${ }^{45}$

Les pronoms de la première personne abondent dans les deux extraits cités ci-dessus. Il convient de préciser que ce discours un peu «nombriliste» ne caractérise pas tous les comptes rendus tirés de la blogosphère et figurant dans notre corpus, loin de là. Mais il existe de toute évidence des blogueurs qui souhaitent s'adresser de façon très personnelle et intime à leurs lecteurs. En conséquence, ils rédigent un type de critique qui, sur le plan du style, se distingue nettement des comptes rendus publiés dans la presse papier. Le côté personnel et intime est aussi accentué par le fait que les blogueuses citées ci-dessus se présentent par leurs prénoms, "Malin» et «Kristin». Il y a ici une différence fondamentale entre la blogosphère et la presse traditionnelle. Sur Internet, l'interactivité entre blogueur et lecteur est un aspect naturel de la communication, alors qu'elle est quasi inexistante dans la presse papier.

44. «Jag vet inte vad det var med den här boken som jag hade så svårt för. Någonstans kände jag att jag aldrig lyckades etablera en relation till karaktärerna. Personerna fastnade inte och jag kunde inte komma ihåg vem som var vem. Det gjorde hela läsningen smått kaotisk, för att inte säga tråkig. Jag hade svårt att hålla koll på vem som var vem och vilken roll de hade. Jag tror att en del böcker ska man läsa vid en viss tidpunkt för att kunna ta dem till sig. Den här boken kanske är en sådan.»

45. «Berättarrösten, den 15 åriga Doria, får mig genast att tänka på lilla Momo i en av mina absoluta favoritböcker La vie devant soi av Romain Gary. Det ger mig blandade känslor. Jag blir upprymd över att få möta något liknande, samtidigt är det inte lätt att leva upp till en favoritbok.» 
Selon Ardelet et Brial (2011, p. 45-69), c'est le sentiment d'affinité psychologique entre l'émetteur du message et les récepteurs qui fait que les blogueurs influencent l'attitude des internautes. Dans ce contexte, l'usage du prénom et le ton subjectif(je-me-moi) et intime employé par les deux blogueuses sont des aspects importants, on le devine, pour créer un tel sentiment d'affinité. Comme nous l'avons mentionné à la section 2.1, les blogueurs jouent un rôle important dans le marché du livre, car, à l'instar des critiques professionnels s'exprimant dans la presse papier, ils bénéficient en général d'un capital de confiance non négligeable auprès de leur public. En tant que "prosommateurs", ils informent leurs lecteurs de l'existence des produits disponibles sur le marché (en l'occurrence des livres) et, en donnant des opinions subjectives, ils sont susceptibles d'influencer le comportement d'achat de leurs lecteurs ${ }^{46}$. Dans le domaine du marketing, le terme «influenceur» est parfois utilisé pour désigner ces personnes, surtout dans le contexte d'Internet et des réseaux sociaux. Il s'agit souvent de blogueurs jouissant d'un grand nombre de lecteurs ou d'abonnés - et rien ne laisse croire que leur influence sur le marché du livre diminuera dans les années à venir.

\section{Conclusion}

En guise de conclusion, nous constatons que l'on traduit assez peu du français vers le suédois - entre 140 et 170 titres sont offerts sur le marché du livre en Suède chaque année, ce qui représente 5 à $6 \%$ de toutes les traductions publiées dans le pays. Néanmoins, tous les romans de Faïza Guène ont été traduits en suédois, ce qui témoigne d'une conviction et d'une persévérance de la part de l'éditeur Norstedt, qui semble vouloir soutenir cette auteure à long terme. Le nombre de comptes rendus relevés dans la presse et la blogosphère indiquent en outre que les ouvrages de Guène ont été non seulement remarqués et commentés par les critiques littéraires de la Suède, mais aussi lus par le public de ce pays.

Les références à d'autres écrivains présentes dans les comptes rendus associent l'œuvre de Faïza Guène à la migrant literature, à la littérature jeunesse et, à un moindre degré, à la littérature prolétarienne. Les opinions exprimées par les blogueurs ne diffèrent

46. Comme nous l'avons vu précédemment, Genette (1987, p. 268-270) parle d'une double fonction d'information et de recommandation, qu'il attribue au préfacier. À notre avis, cela vaut aussi pour les critiques littéraires, tant les professionnels qui écrivent dans la presse traditionnelle que les blogueurs. 
pas de manière significative de celles que l'on trouve dans la presse papier. Leur discours, cependant, s'avère parfois plus subjectif et plus intime que le discours journalistique traditionnel. Nous avons trouvé quelques preuves que le premier roman de l'auteure, Kiffe kiffe imorgon, a été utilisé à des fins didactiques au collège et au lycée en Suède. Cela s'explique peut-être par le fait que l'œuvre de Guène présente des thématiques plus ou moins universelles, susceptibles d'intéresser un grand nombre de jeunes lecteurs, quelle que soit leur origine géographique et socioculturelle - mais surtout, sans doute, ceux qui ont vécu dans une cité de banlieue défavorisée. Or, la pauvreté et l'exclusion sociale décrites par Faïza Guène dans ses romans ne sont pas des phénomènes inconnus en Suède. Dans ce pays où l'on a souvent prôné un État-providence basé sur la solidarité, la cohésion et la sécurité sociale pour tous les citoyens, on se rend compte que les différences socioéconomiques augmentent rapidement depuis quelques décennies et que, dans certaines banlieues, l'ascenseur social est vraiment tombé en panne. Dans ce contexte, l'œuvre de Faïza Guène a trouvé un public non seulement grâce à son humour noir, son langage imagé et ses personnages vivants, mais aussi parce qu'elle décrit une réalité sociale pertinente pour la société suédoise.

\section{Références}

Alakoski, Susanna (2006). Svinalängorna [Les porcheries]. Stockholm, Bonnier.

Ardelet, Caroline et Bérangère Brial (2011). «Influence des recommandations d'internautes : le rôle de la présence sociale et de l'expertise». Recherche et Applications en Marketing, 26, 3, p. 45-69.

Aronsson, Mattias (2012). «La réception sur Internet de Kiffe kiffe demain de Faïza Guène». In E. Ahlstedt et al., dir. Actes du XVIII congrès des romanistes scandinaves/Actas del XVIII congreso de romanistas escandinavos. Göteborg, Acta Universitatis Gothoburgensis, Romanica Gothoburgensia 69, p. 63-80.

Aronsson, Mattias (2015). «Faïza Guène chez les Vikings. Quelques réflexions à propos de la traduction suédoise d'un discours argotique et "beur"». Moderna språk, 109, 1, p. 30-49.

Aronsson, Mattias (2016). «La réception de Marguerite Duras en Suède. La critique professionnelle et non professionnelle». Moderna språk, 110, 2, p. 1-24.

Bakhtiari, Marjaneh (2005). Kalla det vad fan du vill [Appelle-ça comme tu veux, merde]. Stockholm, Ordfront. 
Boyacioglu, Daniel (2003). Istället för hiphop [Au lieu de hip-hop]. Stockholm, Tiden.

Brodow, Ulla (2001). «Datorbaserad kommunikation i språk och interaktivt skrivande [Communication informatique dans les langues étrangères et dans l'écriture interactive] ». In R. Ferm et P. Malmberg, dir. Språkboken [Le manuel des langues]. Stockholm, Skolverket, p. 122140.

Carlsson, Siewert (2010). Skrivarbok. Om konsten att skriva prosa, poesi och journalistik [Manuel sur l'écriture. Sur l'art d'écrire la prose, la poésie et le journalisme]. Västerås, Faun.

Cassirer, Peter (2003). Stil, stilistik \& stilanalys [Style, stylistique \& analyse de style]. Stockholm, Natur och Kultur.

Dixon, Edward et Michael Thomas, dir. (2015). Researching Language Learner Interaction Online. From Social Media to MOOCs. San Marcos, Texas State University, Calico Monograph Series 13.

Doggelito, Dogge et Ulla-Britt Kotsinas (2004). Förortsslang [Argot des banlieues]. Stockholm, Norstedt.

Escarpit, Robert (1970). Le littéraire et le social. Éléments pour une sociologie de la littérature. Paris, Flammarion.

Findahl, Olle (2013). Svenskarna och internet 2013 [Les Suédois et l'Internet 2013]. Stockholm, Stiftelsen för internetinfrastruktur.

Genette, Gérard (1987). Seuils. Paris, Seuil.

Guène, Faïza (2004). Kiffe kiffe demain. Paris, Hachette.

Guène, Faïza (2006a). Du rêtve pour les oufs. Paris, Hachette.

Guène, Faïza (2006b). Kiffe kiffe imorgon. Stockholm, Norstedt.

Guène, Faïza (2008a). Les gens du Balto. Paris, Hachette.

Guène, Faïza (2008b). Drömmar för dårar. Stockholm, Norstedt.

Guène, Faïza (2010). Sista beställningen på Balto. Stockholm, Norstedt.

Guène, Faïza (2014). Un homme, ça ne pleure pas. Paris, Fayard.

Guène, Faïza (2015). En riktig man grăter inte. Stockholm, Norstedt.

Hassen Khemiri, Jonas (2003). Ett öga rött [Un œil rouge]. Stockholm, Norstedt.

Jenkins, Henry (2006). Convergence Culture. Where Old and Nerw Media Collide. New York, New York University Press.

Jenkins, Henry, Mizuko Ito et Danah Boyd (2015). Participatory Culture in a Networked Era. Cambridge, Polity.

Leiva Wenger, Alejandro (2001). Till vår ära [À notre honneur]. Stockholm, Bonnier.

Linderborg, Åsa (2007). Mig äger ingen [Personne ne me possède]. Stockholm, Atlas.

Lindqvist, Yvonne (2005). Högt och lågt $i$ skönlitterär översättning till svenska [Haut et bas dans la traduction littéraire en suédois]. Uppsala, Hallgren \& Fallgren. 
Möijer, Kjell (1989). Svensk språkstil. Stil Eீ stilanalys [Style langagier suédois. Style \& analyse de style]. Solna, Ekelunds.

Nationalbibliografin isiffror 2016[Bibliographie nationaleen chiffres](2016). Stockholm, Kungliga biblioteket - enheten för nationalbibliografin. Disponible à <http://www.kb.se/dokument/Nationalbibliografin\% 20i\%20siffror\%202016/Nationalbibliografin\%20i\%20siffror_2016 slutversion.pdf/> [consulté le 26 octobre 2017].

Olsson, Kenneth (2011). Le discours beur comme positionnement littéraire. Romans et textes autobiographiques français (2005-2006) d'auteurs issus de l'immigration maghrébine. Thèse de doctorat. Stockholms universitet. Disponible à <https://www.diva-portal.org/smash/get/diva2:454828/ FULLTEXT01.pdf> [consulté le 16 janvier 2018].

Ritzer, George (2010). «Focusing on the Prosumer. On Correcting an Error in the History of Social Theory». In B. Blättel-Mink et K.U. Hellmann, dir. Prosumer Revisited. Zur Aktualität einer Debatte. Wiesbaden, Verlag für Sozialwissenschaften, GWV Fachverlage, p. 61-79.

Ritzer, George, Paul Dean et Nathan Jurgenson (2012). «The Coming of Age of the Prosumer».American Behavioral Scientist, 56, 4, p. 379-398.

Sarland, Charles (1991). Young People Reading: Culture and Response. Milton Keynes et Philadelphia, Open University Press.

Steiner, Ann (2012). «Digital litteraturkritik [Critique littéraire informatisée]». In C. Lenemark, dir. Litteraturens nätverk: berättande på Internet [Réseaux de la littérature : narrations sur Internet]. Lund, Studentlitteratur, p. 51-63.

Steiner, Ann (2015). Litteraturen i mediesambället [La littérature dans la société des médias]. Lund, Studentlitteratur.

Svenska förläggareföreningen [Association des éditeurs suédois] (2017). «Bokläsningen [La lecture des livres]». Disponible à < http://www. forlaggare.se/boklasningen-0> [consulté le 9 août 2018].

Säljö, Roger (2015). Lärande. En introduktion till perspektiv och metaforer [Apprentissage. Une introduction aux perspectives et métaphores]. Malmö, Gleerups.

Tornberg, Ulrika (2009). Språkdidaktik [Didactique des langues]. Malmö, Gleerups.

Walpole, Jane Raymond (1974). «Eye Dialect in Fictional Dialogue». Collage Composition and Communication, 25, 2, p. 191-196.

Mattias Aronsson

Département de français

Université de Dalarna

Falun, Suède

mar@du.se 


\section{Annexe : corpus étudié}

9c läser Kiffe kiffe imorgon av den franska författarinnan Fä̈za Guène [La classe 9c lit Kiffe kiffe demain de l'auteure française Faïza Guène] (2012). «Kiffe kiffe imorgon». 26 avril. Disponible à < http://kiffekiffeimorgon. blogspot.se/2012/04/> [consulté le 27 juin 2017].

Adolfsson, Eva (2008). «Drastisk och humoristisk. Fä̈a Guènes Drömmar för dårar blandar skarp samhällskritik med fyndig dialog [Radical et humoristique. Du rêve pour les oufs de Fäza Guène, mélange critique social aigu et dialogues ingénieux]». Dagens Nyheter, Stockholm, 24 mai.

Anonyme (2009). «Kiffe kiffe imorgon [Kiffe kiffe demain]». 11 juin. Disponible à <https://www.boktipset.se/lists/ show_list.cgi? context= member\&type=reviews\&key=8537> [consulté le 27 juin 2017].

Asalun (2008). «[Commentaire, sans titre]». 6 mai. Disponible à <http:// bokhora.se/2006/kiffe-kiffe-imorgon/> [consulté le 27 juin 2017].

Berge, Andrea (2006). "Mig lurar ingen! [Personne ne me trompe!]». 18 septembre. Disponible à < http://dagensbok.com/2006/09/18/faizaguene-kiffe-kiffe-imorgon/> [consulté le 27 juin 2017].

Boktoka (2006). «Kiffe kiffe imorgon av Faïza Guène [Kiffe kiffe demain de Faïza Guène]». 7 juin. Disponible à <https://boktoka.se/?p=96> [consulté le 27 juin 2017].

Bonnevier, Lena (2010). «Bàde sorgligt och lustigt [À la fois affligeant et amusant] ». Värmlands folkblad, Karlstad, 25 janvier.

Busck, Christina (2010). «Sista beställningen på Balto. Faïza Guène [Les gens du Balto. Faïza Guène] ». Ljusnan, Bollnäs, 9 février.

Carlander, Jakob (2015). «Friskt flödande om fördomar [Frais et coulant à propos des préjugés]». Östgöta Correspondenten, Linköping, 11 août.

Chahboun, Naima (2015). «Litterär injektion i den samtida livskroppen [Injection littéraire dans le corps social contemporain]». Expressen, Stockholm, 15 septembre.

Dahlman, Inger (2015). «En berörande roman om integration [Un roman touchant sur l'intégration]». Norrköpings Tidningar, Norrköping, 28 juillet.

Dorai, Dalila (2010). « Roligt och angeläget från ändhållplatsen [Amusant et urgent du terminus]». Hallandsposten, Halmstad, 28 janvier.

Ekelund, Fredrik (2013). «Förnyelse som möter motstånd [Innovations qui se heurtent à la résistance]». Sydsvenskan, Malmö, $1^{\text {er }}$ septembre.

Ekenberg, Kristian (2010). «Jag fattar inte hajpen [Je comprends pas la hype]». Arbetarbladet, Gävle, 15 février.

Eklund, Ivana (2009). "Kiffe-kiffe imorgon [Kiffe kiffe demain]». 25 mars. Disponible à <http://www.bokmania.net/2009/03/kiffekiffe-imorgon.html> [consulté le 27 juin 2017]. 
Enander, Crister (2010). «Litteratur: Fä̈za Guène, Sista beställningen på Balto [Littérature : Faïza Guène, Les gens du Balto]». Tidningen Kulturen, Umeå, 26 janvier.

Eriksdotter Andersson, Li (2015). «Det måste få vara svårt [Il faut accepter les difficultés]». Skånska Dagbladet, Malmö, 29 août.

Falkehed, Magnus (2008). «Guide till förorten [Guide de la banlieue]». Sydsvenskan, Malmö, 8 mai.

Filip Otelea, Mariana (2008). «Skriver lättläst och kritiserar tungt [Prose facile à lire et critique mordante]». Göteborgs Fria, Göteborg, 10 juin.

Flakierski, Gregor (2010). «Hopplöshetens verklighet [La vérité du désespoir]». Allehanda, Örnsköldsvik, 13 mars.

Gordan, Sara (2010). «Bortom betongen [Au-delà du béton]». Dagens Nybeter, Stockholm, 19 janvier.

Hagnell, Anna (2015). «I valet mellan utanförskap eller assimilering [Exclusion ou assimilation, l'embarras du choix]». Sändaren, Stockholm, 22 septembre.

Jennie (2010). «Sista beställningen på Balto av Faïza Guène [Les gens du Balto de Faïza Guène]».10 mars. Disponible à <http://jenniesboklista. $\mathrm{com} /$ ?m=201003> [consulté le 27 juin 2017].

Johansson, Viktor (2008). "Språket brinner. Viktor Johansson läser uppföljaren till en fransk succé [La langue brûle. Viktor Johansson lit la suite d'un succès français]». Aftonbladet, Stockholm, 19 mai.

Jonsson, Lena (2015). «Familj drabbas av svår kris [Famille touchée par grave crise]». Skaraborgs Allehanda, Skövde, 16 septembre.

Karlsson, Elise (2010). «Suverän underhållare [Amuseuse extraordinaire]». Svenska Dagbladet, Stockholm, 18 janvier.

Kjersen Edman, Lena (2008). «[Commentaire, sans titre]». 6 mai. Disponible à <http://bokhora.se/2006/kiffe-kiffe-imorgon/> [consulté le 27 juin 2017].

Kopra, Martina (2006). «Cynisk humor och ständiga undanflykter [Humour cynique et interminables excuses]». Tidningen Kulturen, Umeå, 7 décembre.

Kristin (2014). «Faïza Guène x 2 ». 27 août. Disponible à <http://lasresan. se/faiza-guene-x-2/> [consulté le 27 juin 2017].

Kristina (2015). «Författare: Faïza Guène. Titel: En riktig man gråter inte [Auteur : Faïza Guène. Titre : Un homme, ça ne pleure pas]». Disponible à <http://www.bymarkensmanusmedia.com/ bokrecensioner-2015/> [consulté le 27 juin 2017].

Krönlein, Cecilia (2010). «Kvar i förorten [Arrêtée en banlieue]». Göteborgs-Posten, Göteborg, 25 janvier.

Kvist, Lena (2008). «Häng på och gilla läget [Viens ici et accepte la situation]». Borås Tidning, Borås, 30 mai.

Lindbäck, Johanna (2006). «Kiffe kiffe imorgon [Kiffe kiffe demain]». 
31 août. Disponible à <http://bokhora.se/2006/kiffe-kiffe-imorgon/> [consulté le 27 juin 2017].

Lindqvist, Inga-Lina (2015). « Kompromisslös bild av integration [Image sans fard de l'intégration] ». Aftonbladet, Stockholm, 10 août.

Lunderquist, Thomas (2008). «Uppföljaren når inte samma höjd [La suite n'atteint pas le même niveau]». Svenska Dagbladet, Stockholm, 19 mai.

Malin (2012). «Kiffe kiffe i morgon av Faïza Guène [Kiffe kiffe demain de Faïza Guène]». 7 août. Disponible à <https://malinsblog.wordpress. com/2012/08/07/kiffe-kiffe-i-morgon-av-faiza-guene/> [consulté le 27 juin 2017].

Mattsson Flennegård, Malin (2015). «En familj bortom klichéerna [Une famille au-delà des clichés]». Katrineholms-Kuriren, Katrineholm, 11 août.

Nilsson, Camilla (2010). «Koncis skildring av brokig förort [Narration dense d'une banlieue hétéroclite]». Norrköpings Tidningar, Norrköping, 13 février.

Ohlsson, Lotta (2016). «Kärlek, galenskaper och mystik i boktipsen [Amour, folies et mysticisme dans les livres recommandés]». 24 février. Disponible à <http://web.retriever-info.com/services/archive/search> [consulté le 27 juin 2017].

Pelleskopmatten (2014). «Guène, Faïza: Sista beställningen på Balto [Guène, Faïza: Les gens du Balto]. (2010)». 21 juin. Disponible à <biblioskop.blogg.se> [consulté le 27 juin 2017].

Persson, Annika (2010). «Vem slog ihjäl den hatade barägaren? [Qui a tué le propriétaire de bar détesté?]». Dagens Nyheter, Stockholm, 23 janvier.

Petra (2009). "Kiffe kiffe imorgon [Kiffe kiffe demain]». 30 juin. Disponible à <http://enannansidabok.blogspot.se/2009/06/kiffe-kiffeimorgon.html> [consulté le 27 juin 2017].

Pärssinen, Ingrid (2008). «Förortslivet skildrat med svart humor [La vie de banlieue racontée avec un humour noir]». Sydsvenskan, Malmö, 29 mai.

Risberg, Angelica (2007). «23-åriga Faïza Guènes succéroman Kiffe kiffe imorgon är obligatorisk läsning i franska skolor [Kiffe kiffe demain, le roman à succès de Faïza Guène, 23 ans, est lecture obligatoire dans les écoles françaises]». Svenska Dagbladet, Stockholm, $1^{\text {er }}$ octobre.

Setterwall Klingert, Amanda (2015). «Bagatell med flera bottnar [Bagatelle aux couches multiples]». Upsala Nya Tidning, Uppsala, 25 août.

SP2A Bolandgymnasiet [Classe SP2A, lycée de Boland] (2009). "Kiffe kiffe imorgon [Kiffe kiffe demain]». 15 mai. Disponible à <http:// bokbloggenuppsala.blogspot.se/2009/05/kiffe-kiffe-imorgon-avfaiza-guene.html\#!/2009/05/kiffe-kiffe-imorgon-av-faiza-guene. html> [consulté le 27 juin 2017].

Steinsaphir, Marianne (2007). «Grattis Guène! [Félicitations Guène!]». Aftonbladet, Stockholm, 5 juin. 
Stenberg, Bella (2010). «Diskriminering på deckarvis [Discrimination en guise de polar]». Göteborgs-Posten, Göteborg, 17 janvier.

Stjärtmes (2009). "Drömmar för dårar [Du rêve pour les oufs]». $1^{\text {er }}$ juin. Disponible à <https://ofbooksandbirds.blogspot.se/2009/06/ drommar-for-darar.html> [consulté le 27 juin 2017].

Strängberg, Ulla (2015). «Fördomsfullt om integration [Pleins de préjugés sur l'intégration] ». Värnamo Nyheter, Värnamo, 10 août.

Svensson, Amanda et M. Gourdon (2010). «Sista beställningen på Balto [Les gens du Balto]». Sydsvenskan, Malmö, 12 janvier.

Tunbäck-Hanson, Monika (2008). «Fä̈za Guène: Drömmar för dårar [Faïza Guène : Du rêve pour les oufs]». Göteborgs-Posten, Göteborg, 21 mai.

Viksten, Elin (2010). «Hennes förortsböcker blev succé [Ses romans sur la banlieue sont devenus des succès]». Arbetarbladet, Gävle, 15 février.

Wirdelöv, Johan (2008). «Välkommen till förenklandet [Bonjour les simplifications]». 21 août. Disponible à <http://dagensbok.com/2008 /08/21/faiza-guene-drommar-for-darar/> [consulté le 27 juin 2017]. 